\title{
Alterações hepáticas na doença celíaca
}

\author{
Rita Franca ${ }^{1}$ \\ Daniel R. Diniz-Santos ${ }^{1}$ \\ Daniel Simões $M$ ay ${ }^{2}$ \\ Thais Lima Saback ${ }^{2}$ \\ Luciana Rodrigues Silva ${ }^{3}$
}

\begin{abstract}
Resumo
0 envolvimento do fígado tem sido freqüentemente descrito em pacientescelíacos. Condiçõescomo hipertransaminasemia, que retorna a val ores normais após a dieta isenta de glúten, doenças hepáticas de origem auto-imunee outras doenças crônicas do fígado, sobretudo a hepatite crônica pelo vírus $C$, estão associadas com a doença celíaca. 0 objetivo deste artigo édiscutir as relações recentementeevidenciadas na literatura entreessa enteropatia glúten-sensível eos tipos de alterações hepáticas.
\end{abstract}

Palavras-chave: D oençacelíaca, hepatite auto-imune, hipertransaminasemia.

\section{INT RO DUÇÃO}

U m grande número de pacientes adultos e crianças com D oença Celíaca (DC) apresenta alteraçôes hepáticas. 0 envolvimento do fígado em pacientes celíacos pode ser demonstrado de várias formas; pode haver hipertransaminasemia, que retorna, a valores normais após a dieta isenta de glúten, alterações que configuram uma doença hepática de origem auto-imune, bem como associação com outras doenças crônicas do fígado. Este estudo faz uma revisão dos aspectos descritos até 0 momento e da patogênese nesses tipos de envolvimento do fígado que podem ocorrer na DC.

Em 1990, a partir da revisão dos critérios diagnósticos atualizados para D oença celíaca, estabelecidos pela ESPGAN (Sociedade Euro- péia de Gastroenterologia Pediátrica e N utrição), adotou-se uma abordagem prática e sistematizada para o diagnóstico da doença celíaca. São considerados indispensáveis para o diagnóstico da DC os achados de alterações características na mucosa intestinal em, no mínimo, um espécime de biópsia, achados esses que devem estar associados à presença de marcadores sorológicos positivos, havendo, na evolução do paciente, remissão clínica dos sintomas e negativação da sorologia, quando instituída a dieta isenta de glúten. ${ }^{1}$

A D oença Celíaca apresenta um amplo espectro de manifestações clínicas, o que torna o seu diagnóstico um desafio em muitas situações. As formas clássicas, consideradas estágios

\footnotetext{
${ }^{1}$ D outorando. Programa de Pós-graduação em M edicina e Saúde. Faculdade de M edicina - UFBA. Salvador - BA

${ }^{2}$ Acadêmico. Curso de Graduação em M edicina. Faculdade de M edicina - UFBA. Salvador - BA

${ }^{3}$ Professora Titular de Pediatria. Chefe do Serviço de Gastroenterologia Pediátrica - Faculdade de M edicina - U FBA. Salvador - BA

\author{
Rita Franca. \\ Rua M acapá 461/701 - O ndina. \\ 40.170 - 150 Salvador - Bahia - Brasil. \\ Tel: (71) 3237-1472 \\ Fax: (71) 3331-4669 \\ E-mail: rcfmelo@yahoo.com.br
}

Correspondência para / Careapandance ta
} 
avançados dessa condição, são cada vez mais raras na Europa, tendo sido demonstrado, nos estudos de "screening" populacional, que a maioria dos indivíduos portadores dessa patologia apresenta sintomas sutis e inespecíficos. ${ }^{1}$

$G$ randes avanços com os estudos populacionais e com o desenvolvimento dos marcadores sorológicos, tais como os anticorpos antiendomísio, antigliadina e transglutaminase, permitiram a triagem e o diagnóstico precoce dessa enteropatia conseqüente à exposição ao glúten, muito embora a confirmação desse diagnóstico ainda necessite dos achados histológicos característicos da biópsia do intestino.

$\mathrm{N}$ as formas potenciais e iniciais da doença, os indivíduos podem apresentar marcadores sorológicos compatíveis com a patologia, porém sem alterações na mucosa intestinal na vigência de consumo do glúten, o que, no futuro evolui para o desenvolvimento das alterações histológicas compatíveis com a doença. ${ }^{2} 0$ estudo realizado por Sbarbati e colaboradores ${ }^{3} \mathrm{em}$ 2003 vem a confirmar essa possibilidade: quando eles estudaram esses pacientes potenciais, evidenciaram a possibilidade de detectar alterações mínimas da DC na mucosa intestinal por microscopia eletrônica, não são observadas à luz do microscópio convencional.

O panorama epidemiológico da DC mudou de modo significativo através dos estudos populacionais, ao se demonstrar que a doença celíaca representa uma condição de elevada freqüência ${ }^{4,5}$, sendo cada vez mais importante identificar os pacientes com manifestações iniciais ou aqueles assintomáticos. A DC deve ser investigada em familiares de portadores dessa condição, em pacientes hepatopatas e diabéticos, naqueles com anemia não responsiva ao ferro, em pacientes com alterações do esmalte dentário e naqueles com manifestações psiquiátricas. $6,7,8,9,10$

Apenas $10 \%$ a $20 \%$ dos pacientes apresentam a doença clínica com sintomas clássicos, e a maioria apresenta a doença silenciosa ou oligossintomática. D esde 1980, a maior preocupação tem sido o risco potencial descrito para malignidade nos pacientes com DC, principalmente o Linfoma de H odgkin. Vários estudos recentes, feitos com populações mais numerosas, têm demonstrado que o risco de desenvolver doenças malignas passa a ser igual ao da população geral após a introdução da dieta isenta de glúten. Considerando o impacto significativo dessa dieta em termos de custo e de qualidade de vida, tem sido argumentado por alguns autores que pacientes assintomáticos com D oença C elíaca devem ser tratados. 11, 12, 13

U ma grande variedade de lesões hepáticas, particularmente do tipo inflamatória, tem sido descrita em pacientes com D oença Celíaca. $M$ ais raramente, outras lesões do fígado, como esteatose, cirrose e carcinoma hepatocelular, foram descritas em biópsias hepáticas de crianças e adultos com DC, à época do diagnóstico. ${ }^{7}$

As possibilidades de envolvimento hepático na doença celíaca são apresentadas a seguir.

\section{H ipertransaminasemia}

o comprometimento reversível do fígado e de gravidade variável é bem conhecido em adultos e crianças durante a fase ativa da D oença C elíaca. Um distúrbio moderado da função hepática, caracterizado por el evação de aminotransferases (aspartato aminotransferaseAST e alanina aminotransferase-ALT ) com bilirrubinas e gamaglutamiltransferase normais, foi observado em $42 \%$ dos adultos e $54 \%$ das crianças. ${ }^{14}$

Um estudo realizado em crianças demonstrou que a lesão hepática ocorre não somente com a intolerância ao glúten, mas também com intolerância a outras proteínas alimentares, especialmente quando há lesões moderadas ou graves da mucosa intestinal. Esses achados favorecem a hipótese de que a lesão hepática, na D oença Celíaca, está associada com a lesão da mucosa intestinal e pode ser causada não somente por reações imunes, mas também por substâncias tóxicas endógenas ou exógenas, absorvidas pelo intestino. É possível, também, que a lesão hepática ocorra secundariamente à desnutrição geral ou específica. ${ }^{15}$ Essa situação tem levado alguns autores a estudar a possibilidade de tratamento que envolve 0 restabelecimento da mucosa intestinal, a fim de melhorar as alterações hepáticas. ${ }^{4,7}$ 
$\mathrm{N}$ a maioria dos pacientes, as enzimas hepáticas normalizam dentro de 12 meses de exclusão do glúten na dieta; a persistência desses níveis de aminotransferases alterados, após a introdução da dieta específica, necessitam, contudo, de investigação, devido à presença de le sões hepáticas relacionadas a infecções virais ou doença auto-imune sistêmica. ${ }^{14,16}$

$\mathrm{N}$ os casos que se tem realizado a biópsia hepática, as alterações histológicas são leves e inespecíficas, com hiperplasia das células de Kupffer, infiltrado de células mononucleares, esteatose e fibrose discreta. ${ }^{14}$

\section{D oença hepática auto-imune}

Em adultos, recentemente, foi descrita uma prevalência elevada da D oença Celíaca associada às doenças auto-imunes dos ductos biliares, como a cirrose biliar primária, colangite auto-imune e colangite esclerosante primária. A prevalência de cirrose biliar primária varia de $0,17 \%$ a $3 \%$ em pacientes celíacos, e entre $2 \%$ e $7 \%$ dos pacientes com DC podem ser afetados por cirrose biliar primária. Em crianças, casos esporádicos de $\mathrm{H}$ epatite auto-imune ou de colangite esclerosante têm sido descritos em associação com essa doença. 7, 14, 17, 18

A associação entre Cirrose Biliar Primária e DC foi inicialmente descrita por Logan e colaboradores em 4 pacientes. Eles enfatizaram a importância de se reconhecer que essas duas afecções podem ocorrer simultaneamente e que a perda ponderal, nesses pacientes, pode não ser por esteatorréia secundária à redução da secreção de ácidos biliares, e sim pela enteropatia glúten-sensível. ${ }^{19}$

Existeuma relação entre D oença C elíaca e os genes de histocompatibilidade, especialmente os fenótipos H LA B8, H LA DR3, H LA DR7 e DQW 2. N umerosas doenças têm sido descritas em associação com DC, e a maioria delas é de origem auto-imune, associadas com alguns haplótipos dessa enteropatia. ${ }^{20} \mathrm{~A} \mathrm{He}$ patite auto-imune ( $\mathrm{H} \mathrm{Al})$ e a Doença C elíaca com freqüência compartilham haplótipos $H \mathrm{LA}$ semelhantes (H LA D R3-D Q 2 ou D R4-D Q 8). 21

A H Al parece ser um fator de risco para 0 desenvolvimento de DC e vice-versa. ${ }^{21} \mathrm{~A}$ prevalência de DC em pacientes com H Al varia de $4 \%$ no tipo 1 a $8 \%$ no tipo 2. ${ }^{22,23}$

Volta e colaboradores estudaram 181 pacientes adultos e pediátricos com hepatite auto-imune e encontraram oito pacientes (4\%) com anticorpo antiendomísio positivo do tipo IgA. Cinco desses pacientes apresentaram biópsia de duodeno com atrofia vilositária subtotal, e três deles sem apresentar sintomas gastrointestinais. ${ }^{22}$

Em outro estudo, realizado com 96 crianças portadoras H epatite auto-imune do King's College, em Londres, a prevalência de Doença Celíaca foi significativamente elevada de 3,4\%. ${ }^{23}$ Arvola e colaboradores, em 2002, descreveram o caso de uma menina de 11 anos com DC, tireotoxicose e H epatite auto-imune, apresentando vários familiares afetados com doença auto-imune ou DC. ${ }^{24}$

M agiore e Caprai ${ }^{7}$, em um estudo de re visão sobre a relação das doenças hepáticas e a doença celíaca, conclui que:

- Todos os tipos de doenças auto-imunes podem estar associados com a DC, e a H Al foi a mais freqüentemente encontrada.

- Todos os subtipos de H Al podem estar associados com a DC Tipo I, Tipo II, Tipo III, mesmo a forma sem marcadores imunológicos.

- A deficiência completa de IgA e a pre sença de infiltrado eosinofílico no trato portal são características dos pacientes com H Al e D C.

- A re-introdução do glúten na dieta pode associar-se com a recaída das alterações hepáticas em pacientes sem imunossupressão.

Embora ainda não esteja estabelecido o impacto da dieta isenta de glúten nas crianças portadoras de $\mathrm{HAI}$ e DC, já foi descrito que a dieta pode reverter a insuficiência hepática de adultos em vários tipos de doença crônica do fígado. Tal impacto é difícil de ser avaliado, pois geralmente a terapia imunossupressora é iniciada concomitantemente com a dieta isenta de glúten. 13, 21

$\mathrm{Na}$ Itália, um estudo realizado por Ventura, M aggazu e Greco em 909 crianças com DC demonstrou uma prevalência elevada, 1,1\% de casos com hepatite auto-imune, principalmente naquelas crianças com mais de 3 anos à época do diagnóstico, e concluiu que o desen- 
volvimento dessas doenças auto-imunes em pacientes celíacos está relacionada com o tempo de exposição ao glúten. ${ }^{25}$ Esses dados sugerem que o "screening" para D oença C elíaca deve ser realizado em pacientes com hepatite crônica atípica, em que se suspeita da origem auto-imune...$^{25} \mathrm{~A}$ doença celíaca pode ser silenciosa e somente ser reconhecida após o diagnóstico de hepatite auto-imune. ${ }^{22}$

\section{Associação com doenças infecciosas}

Acredita-se que vírus ou outros agentes infecciosos possam ativar reações imunológicas e desencadear o fenômeno da autoimunidade. Suspeita-se que a infecção crônica pelo virús C da hepatite desencadeie uma resposta celular inflamatória específica ao H LA-D Q $2{ }^{26}$ ligado à $\mathrm{DC}$ e outras doenças auto-imunes. ${ }^{27,28}$

Em 2001, Fine e colaboradores ${ }^{29}$ demonstraram que a frequêencia de $D$ oença C elíaca na forma sub-clínica era maior nos pacientes com hepatite crônica pelo vírus C $(1,2 \%)$, quando comparados com outros portadores de hepatopatias não auto-imunes ( $0 \%$ ) e com indivíduos sadios $(0,4 \%)$; interessante foi o fato de que o único paciente do grupo controle com DC, durante a avaliação posterior, foi diagnosticado como portador da infecção pelo vírus da hepatite C (VHC). Além disso, a associação entre a infecção crônica pelo VHC e outras doenças auto-imunes extra-hepáticas, como crioglobulinemia, liquen plano, tireoidite autoimune, Síndrome de Sjögren, entre outras, já foi estabelecida. 30, 31, 32, 33, 34, 35

$M$ ais recentemente, $D$ urante- $M$ angoni e colaboradores ${ }^{36}$ publicaram um trabalho que novamente identificou uma freqüência de 1,3\% de pacientes com DC assintomática entre os portadores de infecção crônica pelo VHC e de $0,4 \%$ nos controles sadios. Além disso, esse estudo demonstrou um papel importante na ativação da $D C$ durante 0 tratamento com interferon, no qual sete pacientes tiveram o diagnóstico de $D C$, sendo que seis deles já tinham o diagnóstico antes de iniciar o tratamento, embora fossem assintomáticos.

A presença de diarréia, anemia e perda de peso induzidas pelo tratamento com interferon-alfa está significativamente associada com a presença de anticorpo antitransglutaminase positivo antes do tratamento. Assim, todos os pacientes que desenvolvem sintomas sugestivos de DC devem realizar sistematicamente a pesquisa desse anticorpo. $\mathrm{Na-}$ queles em que o diagnostico for confirmado, 0 início da dieta isenta de glúten pode retardar ou evitar a descontinuação do tratamento, fato que parece interferir na resposta virológica sustentada. ${ }^{36}$

M uitos, ainda, são os estudos necessários para aprofundar o conhecimento sobre a doença celíaca e suas relações com as manifestações extra-intestinais, sobretudo as manifestações hepáticas.

0 conhecimento das doenças auto-imunes seguramente ampliará as possibilidades de tratamento e prevenção dessas condições, tais como a doença celíaca, hepatite auto-imune, cirrose biliar primária, diabetes mellitus, tireoidite, que podem representar um grande leque de manifestações que talvez sejam desencadeados precocemente na vida, através de alimentos que lesam a mucosa intestinal.

\title{
Liverdysundionincodiacdise
}

\begin{abstract}
The occurrence of liver dysfunction in patients with celiac disease is frequent. Conditions such as elevated aminotransferases which return to normal values on a gluten free diet, autoimmune liver diseases and other chronic liver diseases, chiefly chronic hepatitis $C$, are associated with celiac disease. This paper aims to discuss the evidence of the interface between celiac disease and that spectrum of liver dysfunction.
\end{abstract}

Keywords Celiac Disease, autoimmune hepatitis, - hypertransaminasemia. 


\section{REFERÊNCIAS}

1 BRAN DT, K.; SILVA, G.A.P. D oença celíaca. In: FERREIRA, C.T.; CARVALHO, E.; SILVA, L.S. (Ed). G astroenterologia e hepatologia em pediatria: diagnóstico e tratamento. Rio de Janeiro: M ED SI, 2003. p.161-174.

2 HILL, I. D. et al. Celiac disease: Working Group Report of The First World Congress of Pediatric Gastroenterology, H epatology and Nutrition. J. Pediatr. G astroenterol. Nutr., Philadelphia, v.35, p.S78-S88, 2002. Suppl.2.

3 SBARBATI, A. et al. Gluten sensitivity and "normal" histology: is the intestinal mucosa really normal? $\mathbf{D}$ ig. Liver $\mathbf{D}$ is., Amsterdam, v.35, n.11, p.768-773, 2003.

4 LAURIN P.; STENHAMMAR, L.; FALTH MAGNUSSON, K. Increasing prevalence of coeliac disease in Swedish children: influence of feeding recommendations, serological screening and small intestinal biopsy activity. Scand. J. G astroenterol., 0 slo, v.39, n.10, p.946-952, 2004.

5 PRATESI, R. et al. Prevalence of coeliac disease: unexplained agerelated variation in the same population. Scand. J. G astroenterol., 0 slo, v.38, n.7, p.747-750, July

2003.

6 HOGBERG, L. et al. Familial prevalence of coeliac disease: a twenty-year follow-up study. Scand. J. G astroenterol., 0 slo, v.38, n.1, p.6165, 2003.

7 MAGGIORE, G.; CAPRAI, S. The liver in celiac disease. J. Pediatr. Gastroenterol. N utr., Philadel phia, v.37, p.117-119, 2003.

8 SUMNIK, Z. et al. High prevalence of coeliac disease in siblings of children with type 1 diabetes. Eur. J. Pediatr., Berlin, v.164, n.1, p.9-12, Jan. 2005.

$9 \mathrm{KALAYCl}, \mathrm{A} . \mathrm{G}$. et al. The prevalence of coeliac disease as detected by screening in children with iron deficiency anaemia. Acta Paediatr., O slo, v.94, n.6, p.678-681, 2005.

10 RAVAGLIA, G. et al. Increased prevalence of coeliac disease in autoimmune thyroiditis is restricted to aged patients. Exp. Gerontol., Tarrytown, v.38, n.5, p.589-595, 2003.

11 VERKARRE, V.; ROM ANA, S.-R.; CERFBEN SUSSAN, N. Glúten-free diet, chromosomal abnormalities, and cancer risk in coeliac disease. J. Pediatr. G astroenterol. N utr., Philadelphia, v.38, p.140-142, 2004.

12 GONZALES-ABRALDES, J. et al. Persistent hypertransaminasemia as the presenting feature of celiac disease. Am. J. Gastroenterol., N ew York, v.94, p.1095-1097, 1999.

13 KAUKINEN, K. et al. Celiac disease in patients with severe liver disease: gluten-free diet may reverse hepatic failure. Gastroenterology, Philadelphia, v.122, n.4, p.881-888, 2002.

14 DAVIDSON, S. Coeliac disease and liver dysfunction. Arch. Dis. Child., London, v.87, p.2893-2896, 2002.

15 LINDBERG, $T$. et al. Liver damage in coeliac disease or other food intolerance in childhood. Lancet, London, n.8060, p.390391, Feb. 1978.

16 BARDELLA, M.T. et al. Prevalence of hypertransaminasemia in adult celiac patients and effect of gluten-free diet. H epatology, Baltimore, v.22, n.3, p.833-836, 1995.

17 LAW SO N , A. et al. Autoimmune cholestitic liver disease in people with celiac disease: a population-based of their association. Aliment. Pharmacol. Ther., Oxford, v.21, p.401-405, 2005.

18 VOLTA, $U$. et al. Celiac disease in autoimmune cholestatic liver disorders. Am. J. Gastroenterol., N ew York, v.97, n.10, p.26092613, 2002.

19 LOGAN, R.F.A. et al. Primary biliary cirrosis and coeliac disease: an association? Lancet, London, n.8060, p.230-233, Feb. 1978.

20 BRIDOUX-HENNO, L. et al. A case of celiac disease presenting with autoimmune hepatitis and erythroblastopenia. J. Pediatr. G astroenterol. N utr., Philadelphia, v.33, n.5, p.616-619, 2001. 
21 IORIO, R. et al. Lack of benefit of glutenfree diet on autoimmune hepatitis in a boy with celiac disease. J. Pediatr. G astroenterol. N utr., Philadel phia, v.39, p.207-210, 2004.

22 VO LTA, V. et al. Frequency and significance of anti-gliadin and anti-endomisial antibodies in autoimmune hepatitis. D ig. D is. Sci., $\mathrm{N}$ ew York, v.43, p.2190-2195,1998.

23 FRAN CAVILLA, R. et al. Coeliac disease in children with autoimmune hepatitis. Dig. Liver D is., Amsterdam, v.33, p.624, 2001.

24 ARVOLA, T. et al. Celiac disease, thyrotoxicosis and autoimmune hepatitis in child. J. Pediatr. G astroenterol. N utr., Philadelphia, v.35, p.90-92, 2002.

25 VENTURA, A.; M AGAZZU, G.; GRECO, L. Duration of exposure to gluten and risk for autoimmune disorders in patients with celiac disease. SIGEP Study Group for Autoimmune D isorders in C eliac Disease. Gastroenterology, Philadelphia, v.117, p.297-303,1999.

$26 \mathrm{CHIANG}$, B.L. et al. Establishment and characterization of N S53 protein-specific T-cell clones from a patient with chronic hepatitis $C$. J. Biomed. Sci., D ordrecht, v.5, p.290-296, 1998.

27 M CCOMBS, C.C. et al. Immune abnormalities associated with HLA-B8: lymphocyte subsets and functional correlates. Clin. Immunol. Immunopathol, O rlando, v.39, p.112-120, 1986.

$28 \mathrm{MICH}$ ALSKI, J.P. et al. HLA-DR, DQ genotypes of celiac disease patients and healthy subjects from the West of I reland. Tissue Antigens, Copenhagen, v.47, p.127-133, 1996.
29 FINE, K.D. et al. Celiac sprue: another autoimmune syndrome associated with hepatitis C. Am. J. G astroenterol., N ew York, v.96, p.138-145, 2001.

30 CLIFFO RD, B.D. et al. High prevalence of serological markers of autoimmunity in patients with chronic hepatitis C. H epatology, Baltimore, v.21, n.3, p.613-619, 1995.

31 AGNELLO, V.; CHUNG, R.T.; KAPLAN, L.M. A role for hepatitis virus infection in type II cryoglobulinemia. N. Engl. J. M ed., Boston, v.327, p.1940-1945, 1992.

32 ADINOLFI, L.E. et al. Epidemiology, clinical spectrum and prognostic value of mixed cryoglobulinaemia in hepatitis $C$ virus patients: a prospective study. Ital. J. G astroenterol., Roma, v.28, n.1, p.1-9, Jan. 1996.

33 PAWLOTSKY, J.M.; DHUMEAUX, D.; $B A G O T, M$. H epatitis $C$ virus in dermatology: a review. Arch. D ermatol., Chicago, v.131, p.1185-1193, 1995.

34 TRAN , A.. et al. High prevalence of thyroid autoantibodies in a prospective series of patients with chronichepatitis $C$ before interferon therapy. H epatology, Baltimore, v.18, p.253257, 1993.

35 RAM O S-CASALS, M. et al. Sjogren's syndrome and hepatitis $C$ vírus. Clin. Rheumatol., Brussels, v.18, p.93-100, 1999.

36 DURANTE-M ANGONI, E. et al. Silent celiac disease in chronic hepatitis c: impact of interferon treatment on the disease onset and clinical outcome. J. Clin. Gastroenterol., N ew York, v.38, n.10, p.901-905, 2004. 


\title{
Intranasel tooth in a peient with deft lipand palate
}

\author{
M iguel Gustavo Setúbal Andrade ${ }^{1}$ \\ Antônio M árcioTeixeira M archionni ${ }^{1}$ \\ M arília Gerhardt de O liveira ${ }^{2}$ \\ Cláiton $\mathrm{H}$ eitz ${ }^{2}$
}

\begin{abstract}
Ectopic eruption into thenasal cavity isa rarephenomenon and even rarer among cleft lip and palate patients. A case of an intranasal tooth associated with cacosmia in a 5-year-old patient with cleft lip and palate is reported. Several previous surgeries had been performed to correct the patient's cleft. The patient was evaluated by computed tomography, and the tooth wasremoved from thenostril. The etiology of this phenomenon is discussed.
\end{abstract}

KeyWords Cleft lip, cleft palate, intranasal tooth, computed tomography

\section{INTRODUCTION}

Supernumerary teeth and ectopic eruption have occurred in several sites within the maxillofacial skeleton ${ }^{1}$. Eruption into the nasal cavity is a very rare phenomenon in normal individuals and even rarer in patients with cleft lip and palate $2,3,4,5$. Intranasal teeth have been described asan inverted mesi odens, deciduous teeth, or permanent teeth growing into the floor of the nasal cavity ${ }^{6,7}$. Their occurrence in patients with cleft lip and palate hasbeen associated with surgeries performed to correct thismalformation. The purpose of this paper is to report a case of an intranasal tooth in a patient with cleft lip and palate and to review the literature. The etiologic, clinical, radiographic, tomographic and histological aspects and treatment for these teeth are discussed.

\section{CASE REPORT}

A five-year old girl was referred by an otolaryngologist to the O ral Surgery Service of the $M$ aster Program on O ral and M axillofacial Surgery and Traumatology at Pontifícia Universidade Católica do Rio Grande do Sul, Porto Alegre, Brazil, for removal of an ectopic tooth from the nasal floor. She presented with a hard mass located inside her right nostril and associated with cacosmia. There was no report of chronic obstruction, rhinorrhoea, epistaxis or purulent discharge. $\mathrm{Her}$ medical history was significant for a right congenital cleft lip and palate for which seven previ oussurgical correcti veprocedureshad been performed. N o other systemic di sorder was reported.

An intra-oral examination showed a deciduous dentition with displacement of the right

\footnotetext{
${ }^{1}$ Professor M estre de Cirurgia e Traumatologia Bucomaxilofacial. Faculdade de O dontologia - FBDC. Salvador - BA

2 Professor D outor. Programa de Pós-graduação em Cirurgia e Traumatologia Bucomaxilofacial - PUC/RS. Porto Alegre - RS
}

Correspondência para / Correspondence to:

M iguel Gustavo Setúbal Andrade

Avenida Silveira M artins 3386 - Cabula

41.150-100 Salvador - BA - Brasil

Telefax: (71) 22085859

E-mail: miguelsetubal@hotmail.com 